CHRISTINe LOMBEZ

ORCID: 0000-0002-0788-2312

Université de Nantes - Institut Universitaire de France christine.lombez@univ-nantes.fr

\title{
L'AFRIQUE DU NORD, UN NOUVEAU CENTRE LITTÉRAIRE FRANÇAIS ENTRE 1940 ET 1944 ? L'EXEMPLE DE TUNISIE FRANÇAISE LITTÉRAIRE AU MIROIR DE LA TRADUCTION
}

La période de l'Occupation allemande en France à partir de l'été 1940 a provoqué un brutal reclassement des valeurs littéraires et une redéfinition des notions de « centre » et de " périphérie » dans la République française des Lettres. Les conséquences de ce phénomène sont particulièrement intéressantes à observer en Afrique du Nord pendant les années 1940-1944. En effet, aussi bien en Algérie avec la revue Fontaine (dirigée par Max Pol Fouchet) qu'à Tunis avec Tunisie française littéraire (pilotée par Armand Guibert et Jean Amrouche), se lit la volonté de reprendre le flambeau moral d'un centre parisien désormais déconsidéré pour créer de nouvelles « capitales littéraires » en marge de la métropole. On étudiera, en se fondant sur Tunisie française littéraire, une publication dont le rayonnement en Afrique du Nord fut conséquent pendant la guerre, le rôle de médiateurs culturels de revues littéraires géographiquement " périphériques », et de ses acteurs dans leur tentative de redéfinir les contours du « centre » (Paris) et de la "périphérie » (l'Empire colonial français) - une initiative où les traductions, notamment de la littérature autochtone, vont se révéler un enjeu d'importance. 


\section{BRÈVE PRÉSENTATION DE TUNISIE FRANÇAISE LITTÉRAIRE DANS LE CADRE DU PROGRAMME TSOcc}

Le programme international « Traductions sous l'Occupation » (TSOcc ; $<$ www.tsocc.univ-nantes.fr>) s'est donné pour but, entre 2014 et 2019, l'identification des œuvres traduites en français durant la période de l'Occupation en France et en Belgique (1940-1944), le dépouillement de manière extensive des publications (toutes orientations confondues) qui s'en sont fait l'écho en France (à Paris, mais aussi en province et outre-mer) et en Belgique occupées, la reconstitution de la trajectoire des médiateurs (éditeurs, traducteurs, enseignants, poètes, etc.), et l'analyse des discours tenus sur la traduction durant les années 1940-1944. Au centre des activités de l'équipe TSOcc pendant cinq ans, ces investigations ont apporté des éléments de grand intérêt (et la plupart encore inédits à ce jour) pour une compréhension plus fine des échanges littéraires en France et dans l'espace francophone à un moment particulièrement délicat de son Histoire ${ }^{1}$. La prise en compte des traductions parues dans les périodiques, jusqu'ici restées en majeure partie invisibles car non répertoriées dans les catalogues bibliographiques, a été un volet important de ces travaux qui ont conduit à étoffer considérablement le nombre de traductions publiées durant la période de référence, nombre de traductions étant parues en France dans des périodiques (à la différence de la Belgique où la situation est exactement inverse). Si certains titres bien identifiés ont pu être consultés dans différentes bibliothèques françaises et belges, d'autres, comme Tunisie française littéraire, ont donné plus de fil à retordre. En effet, c'est indirectement (par des comptes-rendus ou des mentions dans la presse de l'époque) que nous avons découvert l'existence de cette feuille, non répertoriée à la Bibliothèque nationale de France ni dans les Archives de l'Outre-Mer situées à Aix-en-Provence. C'est finalement aux Archives Diplomatiques de Nantes qu'il a été possible de la consulter dans son intégralité.

Journal fondé en 1892 par Victor de Carnières (un juriste catholique, fervent opposant du protectorat et partisan de l'annexion de la Tunisie à la France), La Tunisie française est initialement un quotidien porte-parole de la voix des

${ }^{1}$ Cf. notamment le numéro 8 de la revue Atlantide intitulé : 1943 en traductions dans l'espace francophone européen, C. Lombez (dir.), Université de Nantes, 2018 (consultable en ligne : <http:// atlantide.univ-nantes.fr/-1943-en-traductions-dans-1-espace->) ; C. Lombez (dir.), Traduire, collaborer, résister. Traducteurs et traductrices sous l'Occupation, Presses de l'Université François Rabelais de Tours, coll. « TraHis », 2019 ; C. Lombez, « Political (mis)use of translation: poetry pseudotranslations in Occupied France (1940-1944) », Canadian Review of Comparative Literature 44.4, 2017; C. Lombez, « D'une anthologie à l'autre : que transmettre de la poésie allemande pendant/après l'Occupation? », [dans :] I. Poulin (dir.), Traduction et partages : que pensons-nous devoir transmettre?, Actes du XXXVI ${ }^{\mathrm{e}}$ Congrès de la Société Française de Littérature Générale et Comparée (SFLGC), 2013 (consultable en ligne : <http://sflgc.org/acte/christine-lombez-dune-anthologie-a-lautre-quetransmettre-de-la-poesie-allemande-en-francais-pendant-apres-loccupation/>). 
colons, au tirage modeste (6000 exemplaires en 1943), qui a son siège à Tunis. Orienté politiquement à droite dès ses origines, il suit durant les années de la Seconde Guerre Mondiale une ligne pro-Vichy. À partir du 16 novembre 1940, une page littéraire hebdomadaire paraissant le samedi vient s'insérer à la page 3 du journal, visant à rendre compte de la vie culturelle et intellectuelle française et tunisienne. Cette page où l'on relève notamment les noms déjà connus d'Armand Guibert (qui semble avoir été à l'origine de cette publication) ${ }^{2}$ et de Jean Amrouche, accorde, parmi tous les genres littéraires, une attention soutenue à la poésie, son rôle et sa mission. On y trouve également diverses traductions qui donnent lieu à débats. Dès la première page du 16 novembre 1940 apparaissent les trois signatures phares de la feuille : Armand Guibert, Jean Amrouche et Marcel Sauvage. Une revue rapide ( $c f$. annexe) de quelques articles parus entre novembre 1940 et la fin juin 1942 (moment où Tunisie française littéraire s'interrompt définitivement) permet de se convaincre de la diversité des contributions à la page littéraire de La Tunisie française, qui vont d'articles consacrés à la littérature et à l'art (peinture, musique), à des considérations sur la philosophie de l'Inde, le maréchal Pétain, la psychanalyse ou encore la cuisine française (article de Gertrude Stein). On remarque cependant un intérêt constant accordé à la poésie et sa vocation pendant toute l'existence de Tunisie française littéraire ("Les poètes à l'honneur », « Voici l'heure des poètes », « Mission du poète », « Sainteté de la poésie », etc.) qui fait alors écho à une tendance forte que l'on trouve également dans d'autres revues/périodiques paraissant en métropole (Cahiers du Sud, Poésie) mais aussi dans l'Empire (Fontaine) durant la guerre. Ainsi que l'écrit Sauvage :

Cette mission du poète, elle est simple cependant et de nécessité vitale. Elle consiste à éveiller en nous, dans le secret de nous-mêmes, des résonances légères ou profondes qui nous conduisent de plus en plus loin comme d'écho en écho, vers la connaissance intuitive du monde ${ }^{3}$.

Tunisie française littéraire a une dimension locale assumée, avec des chroniques consacrées à la poésie arabe, kabyle, berbère (on notera la collaboration du poète martiniquais Emmanuel Flavia Leopold qui se fait notamment le médiateur de la poésie créole). Le critique et poète Amrouche défend à plusieurs reprises la possibilité d'une poésie africaine, s'interrogeant

à quelles conditions doit satisfaire une poésie africaine de langue française pour être digne du nom de poésie. À ma connaissance, un seul écrivain jusqu'ici les a aperçues avec une certaine clarté. Les autres [...] n'ont chanté l'Afrique que dans ses aspects extérieurs ${ }^{4}$.

${ }^{2}$ Cf. ici M. Corriou, Les Français et la vie culturelle en Tunisie durant la Seconde Guerre Mondiale, [thèse de l'École Nationale des Chartes], 2005, p. 512.

3 M. Sauvage, « Mission du poète », 23.11.1940. [Toutes les citations sont issues du journal Tunisie française littéraire].

4 J. Amrouche, "Pour une poésie africaine », 5.04.1941. Jean Amrouche fait ici allusion à Jean Joseph Rabearivelo, poète malgache, publié dans les Cahiers de Barbarie d'A. Guibert en 1935. Il fustigera ailleurs « Les maux des cousins de René, tout pétris de littérature, [qui] prennent 
Le problème du bilinguisme de nombreux auteurs est même qualifié de « drame » dans la mesure où, par rapport aux langues autochtones, « tout le charnel et tout le spirituel de la langue lui demeurant dans une grande mesure étranger, l'Africain ne peut guère en user que comme un outil intellectuel » ${ }^{5}$, c'est-à-dire sans une entière adhésion de son être. Amrouche formule le vœu qu' « Il viendra un jeune poète ", qu'un " poète africain se revête de l'Afrique [...], que l'Afrique cesse d'être une idée ou un décor pour être éprouvée du dedans $[\ldots]{ }^{6}$; à ce poète incombera également de

fondre dans un brasier unique ce qui est spécifiquement français et ce qui est spécifiquement africain. Alors un chant purement français et authentiquement africain accomplira le miracle d'unir deux mondes irréductibles ${ }^{7}$.

Corollairement émergent les sujets de la décentralisation culturelle et du régionalisme (probablement liés aux activités de l'association "Jeune France », subventionnée par Vichy avant d'être interdite en 1942) comme effet collatéral de la Débâcle. Guibert salue ainsi avec force la redistribution des cartes des lieux d'influence littéraire, avec l'idée sous-jacente (une position alors également défendue par André Gide) que la renaissance peut seule venir d'Afrique du Nord :

Un Paris-Moloch dévorait, absorbait tout [...]. Il a fallu le désastre pour que la France découvrît ses provinces, leur opulence, leur vertu inspiratrice [...], et par-delà les provinces, ces terres d'Empire qui en sont le prolongement naturel. [...] Décentraliser, c'est en réalité multiplier les centres au détriment de celui qui menaçait de les éclipser tous. [...] Il faut [...] établir des échanges, grouper des vivants par les vivants, et faire rayonner une Tunis africaine, méditerranéenne et humaine, afin qu'on puisse la saluer un jour comme une capitale des lettres et des arts ${ }^{8}$.

Enfin, une ligne nettement pro-Vichy est perceptible (avec des articles sur Charles Péguy, Mgr de Bollon — le « Bossuet algérois » —, Émile Ripert, Grand Majoral du Félibrige et frère de Georges, proche de Pétain —, sur Pétain, Barrès, Brasillach, etc.). Le procès des intellectuels, abordé à plusieurs reprises ${ }^{9}$, est également une thématique vichyste, tout comme celle du redressement que l'on

l'Afrique pour une scène où leurs petites passions se jouent dans un décor grandiose et solitaire » (J. Amrouche, «L'Afrique aussi est une personne », 26.04.1941).

5 J. Amrouche, « Pour une poésie africaine », 19.04.1941.

6 J. Amrouche, « Pour une poésie africaine », 3.05.1941.

7 Ibidem.

8 A. Guibert, « Tunis capitale », 4.01.1941. Cf. également du même auteur « Tunis et la décentralisation littéraire. Regard sur le passé » (28.12.1941).

${ }^{9}$ Cf. ici « Les intellectuels du monde qui appartiennent au monde qui meurt, [...] s'étaient peu à peu enfermés dans la stérile contemplation des constructions abstraites qu'enfantait leur esprit. [...] À force de vouloir vivre dans un monde d'idées pures, ces Byzantins de l'Occident avaient cessé de penser et de sentir le monde » (J. Amrouche, "Procès des intellectuels », 16.11.1940); « être un acteur dans l'immense action dramatique qu'est la Rédemption du monde » (J. Amrouche, «Procès des intellectuels. Qui veut sauver sa vie la perdra », 7.12.1940). 
retrouve sous la plume d'Amrouche affirmant, dans un article sur la « Dignité de la critique », qu'il faut " sauver les valeurs permanentes qui sont notre plus précieux héritage » et ajoutant " c'est notre contribution, modeste, mais que nous croyons nécessaire à ce redressement intellectuel à quoi le Maréchal nous a expressément invités » ${ }^{10}$. Il n'est sans doute pas anodin non plus que dans un article de La Tunisie française du 7.10.1940 (avant l'apparition de Tunisie française littéraire donc) intitulé " Poètes et hommes d'action » soient cités les propos d'Abel Bonnard, collaborateur notoire et futur ministre de l'Éducation nationale (à partir de 1942), se demandant « quel rôle le sentiment poétique doit jouer dans la vie nationale d'un peuple ». On remarquera par ailleurs qu'à partir de juillet 1940, La Tunisie française fait figurer en sous-titre une célèbre phrase du maréchal Pétain (« Je hais les mensonges qui vous ont fait tant de mal »), extraite de son discours du 25.06.1940 annonçant aux Français les conditions de l'armistice.

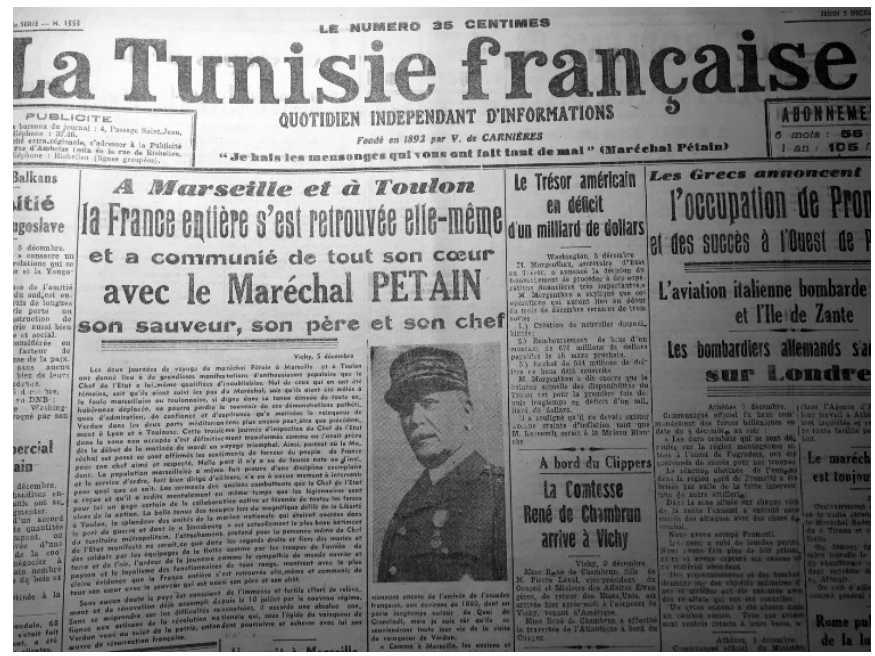

Photo 1. Première page de La Tunisie française du jeudi 5 décembre 1940 (archives de l'auteure)

On ne saurait donc mieux signaler sous quel patronage idéologique se place le journal. En dépit de cela, des personnalités très diverses vont se croiser dans Tunisie française littéraire durant les deux années de son existence. On y trouvera ainsi les signatures de Pierre Seghers, Gertrude Stein, Francis de Miomandre, Henri Bosco ou encore Jean de Boschère. Et celle d'Albert Camus qui ne sera pas des moindres.

10 J. Amrouche, « Dignité de la critique », 1.02.1941. 


\section{TROIS ACTEURS PRINCIPAUX \\ DE LA PAGE LITTÉRAIRE DU JOURNAL}

Même si de nombreux contributeurs participent à l'aventure de Tunisie française littéraire, trois noms reviennent de manière particulièrement fréquente : ceux d'Armand Guibert, de Jean Amrouche et de Marcel Sauvage (ce dernier surtout dans les débuts).

Armand Guibert (1906-1990), natif de Haute-Garonne, poète et écrivain, traducteur de l'anglais, l'espagnol et du portugais. Il fonda en 1934 à Tunis les Cahiers de Barbarie et fut l'un des premiers traducteurs français de Lorca en 1935 (dans le recueil des Chansons gitanes). Il co-anima ensuite avec Amrouche (jusqu'en 1941, date de son départ au Portugal) la page littéraire de La Tunisie française, dans laquelle publieront, entre autres, Pierre Emmanuel, Henri Bosco, Albert Camus et Jules Roy. Si ses collaborations à Tunisie française littéraire portent essentiellement sur la poésie, on y trouve également des articles sur la littérature et la culture portugaises, James Joyce (mort en 1941), mais aussi, Robert Brasillach (une connaissance d'avant-guerre), Charles Péguy ou Henri de Montherlant, plus en phase avec la ligne politique du journal. Il est, comme on l'a vu, un fervent défenseur d'une décentralisation littéraire en direction de l'Afrique du Nord, notamment vers la Tunisie, alors protectorat français.

Jean Amrouche (1906-1962), « algérien universel » (Réjane Le Baut), est un chrétien d'origine kabyle ; collaborateur de Fontaine et de Quatre Vents (deux périodiques publiés à Alger), directeur de la revue L'Arche à partir de février 1944, il est lié avec le précédent depuis les années d'avant-guerre et ses années d'enseignement en Tunisie ; il prend les commandes de Tunisie française littéraire à partir d'avril 1941 au départ de Guibert. Il traduit du kabyle et publiera un volume de Chants berbères. Collaborateur très actif de Tunisie française littéraire tout au long de ses deux ans d'existence, il signe de très nombreux articles portant sur le rôle des intellectuels et celui de la critique, sur la poésie africaine et le folklore berbère, mais également sur Guillaume Apollinaire, Arthur Rimbaud, Stéphane Mallarmé, Pierre Jean Jouve, Jules Roy, Pierre Emmanuel... Il s'intéresse également à la poésie de langue d'oc (troubadours) et au Félibrige.

Marcel Sauvage (1895-1988), homme de lettres et journaliste, lauréat du Prix Max Jacob, est l'initiateur de la page littéraire de Tunis Soir (Tunis-Soir littéraire et artistique), dans laquelle Amrouche voyait une concurrente de Tunisie française littéraire. Traducteur de l'italien, il signe dans Tunisie française littéraire plusieurs articles sur la poésie et les poètes, mais aussi un article hagiographique sur le Maréchal Pétain et sur la « race » française. Ses collaborations, de régulières au début, s'espacent peu à peu à partir de 1941. 
Comme on peut le constater dans cette brève présentation biographique, ces trois rédacteurs ont des origines et des profils on ne peut plus disparates. Ils ont néanmoins un point commun : ils pratiquent la traduction littéraire, sujet dont l'importance va se confirmer au fil des pages de Tunisie française littéraire.

\section{L'INTÉRÊT POUR LA TRADUCTION DANS TUNISIE FRANÇAISE LITTÉRAIRE}

On a déjà noté plus haut l'intérêt porté par Tunisie française littéraire à la poésie, qu'illustrent à diverses reprises de nombreux articles. Pour reprendre les propos du critique André Rousseaux à l'issue d'un voyage à Alger en 1942, « beaucoup plus sûrement que la circulation des agrumes, la circulation de la pensée et de la poésie est aujourd'hui un des liens de l'Empire $»^{11}$.

Plus généralement, l'importance accordée à la poésie durant la guerre pose question, car on serait tenté de penser que devant les urgences et le tragique de l'heure (violences, arrestations, déportations, exécutions), les mots, fussent-ils des poètes, n'ont que peu de poids à opposer. Une raison plus profonde de cet engouement pour la poésie est donnée par Guibert lorsqu'il écrit : " Poètes, votre heure vient $[\ldots]$; au milieu de la souffrance du monde, votre voix est entendue parce qu'elle monte des profondeurs élémentaires de l'être $»^{12}$.

La mission rédemptrice de la poésie en temps de détresse semble bien d'ailleurs un leitmotiv de l'époque ; ainsi, au printemps 1942, à Alger, la revue Fontaine de M.P. Fouchet fait paraître un numéro intitulé « La poésie comme exercice spirituel ». De même, dans les pages du Figaro, P. Seghers fera l'éloge de la poésie, « le langage de la divine proportion », insistant sur le « devoir social » des poètes, et ajoutant : « en ce moment de bestialité majuscule, [il est bon] de considérer le problème de la création poétique comme celui de la recherche de la plus haute harmonie $»^{13}$.

Cependant, l'attention portée à la poésie dans Tunisie française littéraire ne concerne supprimer pas uniquement les poètes français, même s'ils restent numériquement majoritaires au fil des pages. Elle touche également les questions de traduction. Dans la perspective d'un déplacement du centre métropolitain vers la périphérie littéraire nord-africaine, on trouve ainsi une ouverture intéressante à la poésie en langue autochtone. Dès les premiers numéros de Tunisie française littéraire, un intérêt manifeste est marqué pour la création poétique africaine populaire, comme en témoigne cette traduction en vers d'un chant bédouin ${ }^{14}$ :

\footnotetext{
11 A. Rousseaux, « La critique en voyages », Le Figaro littéraire, 23.05.1942.

12 A. Guibert, « Les poètes à l'honneur », 11.10.1940, p. 2.

13 P. Seghers, « Le langage de la divine proportion », Le Figaro, 17.02.1942, p. 3.

14 «Un chant bédouin » par Kh. Abdul Wahab, 25.01.1941.
} 


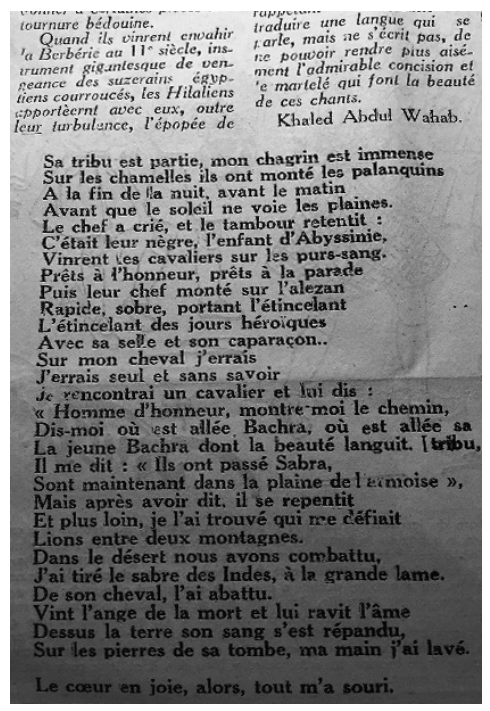

Photo 2. «Un chant bédouin » traduit par Khaled Abdul Wahab, Tunisie française littéraire du 25 janvier 1941 (archives de l'auteure)

On trouve également un article consacré aux poèmes touaregs ${ }^{15}$ ou encore aux chants de danse de Kabylie ${ }^{16}$, cités comme exemples de poésie primitive (au sens « romantique » que put avoir le terme). Dans ce dernier cas, le traducteur, Amrouche, souligne que ces chants « peignent sur le vif les sentiments d'un peuple qui a su garder toutes les grâces de l'enfance : la pureté de regard, la vivacité vierge de la sensation, le don de l'émerveillement $\gg 17$.

Les autres auteurs étrangers qui apparaissent dans Tunisie française littéraire reflètent des affinités propres aux différents chroniqueurs. Guibert signe ainsi plusieurs contributions sur la poésie portugaise accompagnées de traductions en vers (en plus de différents papiers consacrés généralement au Portugal et au monde lusophone qu'il connaît bien) : c'est le cas de « Trois poëmes d'Adolfo Casais Monteiro ${ }^{18}$ ou d' $"$ Un poëme de F. Pessoa », un auteur appelé à une grande fortune littéraire mais encore quasiment inconnu ${ }^{19}$. De même on trouve le nom de Pierre Darmangeat ${ }^{20}$, hispaniste distingué, très actif durant la guerre en zone sud (il collabore entre autres à la revue Pyrénées, aux Cahiers du Sud ou à la revue Méridien), qui signe la traduction (une en vers et deux en prose) de « Trois poèmes de Manuel Machado » (frère d'Antonio) en accompagnement de l'article « À pro-

15 « Poèmes touaregs », recueillis par L. Audouin-Dubreuil, 22.03.1941.

16 «Trois chants de danse du pays kabyle» par J. Amrouche, 27.09.1941.

17 Ibidem, p. 3.

18 «Trois poëmes d'Adolfo Casais Monteiro » par A. Guibert, 20.12.1941.

19 « Un poëme de F. Pessoa » par A. Guibert, 16.05.1942.

${ }^{20}$ Cf. C. Lombez, « Pierre Darmangeat (1909-2004). L'Espagne poétique au cœur », [dans :] eadem (dir.), Traduire, collaborer, résister... 
pos de M. Machado ou actualité de l'inactuel $»^{21}$. Amrouche traduit aussi pour sa part de la poésie italienne ${ }^{22}$. C'est donc surtout des traductions d'écrivains de langues romanes (portugais, espagnol, italien) qui intéressent les rédacteurs, sans doute du fait de leur relative proximité avec Tunis, et leur ouverture sur le bassin méditerranéen.

L'intérêt des rédacteurs se porte également sur les conditions d'importation de la poésie étrangère en français, autrement dit sur la traduction, sa possibilité et sa valeur. Dans «L'expression poétique et le pouvoir des mots », le poète martiniquais et collaborateur à Tunisie française littéraire Emmanuel Flavia Leopold, reprenant des propos d'Edmond Jaloux parus dans Fontaine, souligne que « la prétendue puissance incantatoire des mots passe dans les traductions des grandes œuvres [...] alors qu'elle est absente de millions de vers français sans poésie ${ }^{23}$. Ce point de vue iconoclaste sur la traduction poétique est suffisamment rare pour être signalé. À propos de la traduction des « Chants bédouins », Khaled Abdul Wahab souligne (en s'en excusant presque) « la difficulté de traduire une langue qui se parle mais ne s'écrit pas $»^{24}$. Quant à Guibert, évoquant la traduction publiée à Alger par Charlot du Livre de la pauvreté et de la mort ${ }^{25}$, il fait l'éloge de «M.A. Adamov, à qui nous devons la préface et la traduction de l'ouvrage, toutes deux excellentes ».

Une amorce de lecture «traductologique » comparatiste voit également le jour à diverses reprises, que ce soit dans la revue des revues de Tunisie française littéraire (où un compte-rendu bien informé d'un numéro des Cahiers $d u$ Sud fait mention de diverses traductions de Lorca) ${ }^{26}$, ou encore dans un article d'Amrouche évoquant la nouvelle traduction du Romancero de Lorca par Félix Gattégno $^{27}$. De manière intéressante, ce dernier procède à une mise en perspective de cette version et de celle, plus ancienne, déjà donnée en 1935 par son complice Guibert dans les Chansons gitanes (réalisée en collaboration avec le frère de Lorca, Francisco, alors vice-consul d'Espagne à Tunis). Décrivant l'émerveillement qu'il avait alors éprouvé à voir surgir l'univers chatoyant de Lorca dans ces « chansons » françaises, il a sur la version de Gattégno des propos sans appel, l'accusant même d'avoir trahi le poète espagnol :

21 « Trois poèmes de M. Machado », trad. P. Darmangeat, 26.04.1941.

22 « Haute est la lumière » d'A. Corpora, traduit de l'italien par J. Amrouche, 9.05.1942.

23 E.-F. Leopold, «L'expression poétique et le pouvoir des mots », 20.06.1942.

${ }^{24}$ Kh. Abdul Wahab, « Chants bédouins », 25.01.1941.

25 A. Guibert, « Rilke vous parle», 14.06.1941. A. Guibert souligne que Rilke, « qui éprouvait à l'extrême de sa sensibilité les mouvements de la vie profonde, n'aurait pu survivre à l'époque présente ».

26 «Les revues », 15.03.1941.

27 J. Amrouche, «Une nouvelle traduction de F. Garcia Lorca », 18.10.1941. Sur cette étonnante réception de Lorca durant la période de l'Occupation, voir également C. Lombez, « La réception de la poésie espagnole traduite en France sous l'Occupation (1940-1944) : le cas de Lorca et de ses traducteurs », Revue de Littérature comparée 4, 2019. 
Je ne ferai pas au nouveau traducteur de Lorca, M. F. Gattégno, une mauvaise querelle de grammairien, encore que l'une des premières conditions à laquelle doit satisfaire une traduction soit d'être écrite en français. M. Gattégno, emporté sans doute par l'enthousiasme que chauffait en lui le texte espagnol a laissé dans sa version française nombre de tâches qui trahissent ou bien la hâte ou bien l'incompétence. La version d'un poème ne peut être qu'un autre poème. [...] Certains traducteurs paresseux, escomptant le choc des tournures et des images étranges, [...] se tiennent satisfaits d'une traduction d'autant plus barbare qu'elle est en apparence plus fidèle à l'original. [...] Il n'est pas de plus sûre manière de trahir le poète dont on prétendait servir la gloire ${ }^{28}$.

À la critique du choix d'une traduction à visée sourcière (« le choc des tournures et des images étranges ») se superpose en fait ici un autre argument, dont on sent qu'il est décisif, celui de la supériorité poétique d'une traduction quand elle est réalisée par des poètes : «Il est vrai que les Chansons gitanes ont été traduites par des poètes ${ }^{29}$. Le romancero gitan cherchait un poète français, il n'a rencontré qu'un traducteur $»^{30}$. On ne saurait mieux exprimer le préjugé favorable dont jouit, pour Amrouche, la traduction poétique quand elle est l'œuvre de poètes (ce qui était le cas de Guibert et de lui-même), au détriment de celle faite par de simples professionnels comme Gattégno (qui ne sont « que » traducteurs) qui se retrouvent, sous sa plume, singulièrement dévalorisés.

Une telle activité de lecture comparatiste de traductions en pleine guerre a de quoi laisser songeur. Pourtant, si on y réfléchit davantage, rien n'est plus révélateur que cet exercice de liberté de lecture (toute traduction étant par essence une interprétation dont le sens est relatif, il n'y a pas de traduction absolue ni parfaite) dans un contexte de censure et de discours propagandistes orientés. Comparer et discuter les mérites de plusieurs traductions d'un même texte, n'est-ce pas aussi s'interroger sur les mécanismes de la construction du sens (voire sur le sens luimême), apercevoir qu'il peut être multiple dans une période où le langage, déformé par les idéologies, avait plutôt tendance à n'indiquer qu'une seule direction possible ? Cela est d'autant plus plausible que l'intérêt affiché concernait principalement la traduction poétique et que la poésie, surtout en temps de contrainte, est appréciée pour son caractère " ésopique ${ }^{31}$ permettant le double, voire le triple sens (références cryptées, possibilité de coder des messages ${ }^{32}$ en faisant dire subtilement autre chose au texte, etc.). Pour reprendre ici les mots de Paul Ricoeur, «c'est fou — c'est le cas de le dire — ce qu'on peut faire avec le lan-

28 J. Amrouche, « Une nouvelle traduction... ».

${ }^{29}$ Il s'agit entre autres de Jean Prévost, Jules Supervielle et Armand Guibert lui-même.

30 J. Amrouche, «Une nouvelle traduction... », p. 3.

31 On pense ici au cas du poète et traducteur russe Boris Pasternak qui utilisait la traduction poétique de manière oblique pour déjouer la censure soviétique (cf. C. Lombez, La Seconde Profondeur. La traduction poétique et les poètes traducteurs en Europe au XXe siècle, Les BellesLettres, coll. «Traductologiques », Paris 2016).

${ }^{32}$ L'exemple le plus emblématique ici étant le poème Liberté de P. Éluard, autorisé par un censeur peu scrupuleux qui n'était pas allé plus loin que les premières strophes et avait conclu qu'il s'agissait d'un énième poème d'amour... Il fut publié sous le titre « Une seule pensée » en juin 1942 dans la revue résistante Fontaine. 
gage : non seulement dire la même chose autrement, mais dire autre chose que ce qui est $»^{33}$. La «propension du langage à l'énigme, à l'artifice, à l'hermétisme, au secret ${ }^{34}$ qu'il évoque dans la même page est sans doute l'une des raisons expliquant l'engouement pour la poésie et pour sa traduction durant les années de guerre, deux expériences en acte des « inquiétantes contrées de l'indicible » ${ }^{35}$, un indicible (pour ne pas parler ici d'intraduisible) qui, par définition, résiste à toute tentative de manipulation.

\section{CONCLUSION}

Même si son existence fut brève, Tunisie française littéraire, bien que située à la périphérie du monde des Lettres françaises, sut se positionner comme un centre intellectuel où sont venues débattre des sensibilités souvent diamétralement opposées (Camus affirmant que « le sabre est toujours vaincu par l'esprit » ${ }^{36}$ et Sauvage, faisant dans un article hagiographique l'éloge du sourire du Maréchal Pétain ${ }^{37}$ ). Selon Morgan Corriou,

la vie littéraire tunisienne et plus largement nord-africaine, grâce à la diffusion de Tunisie française littéraire, est marquée par le développement nouveau de pages de grande qualité. Tunisie française littéraire devient une référence. Celle-ci, par son rythme hebdomadaire, suscite dans l'intellectualité d'Afrique du Nord un mouvement constant ${ }^{38}$.

Camus complimentera d'ailleurs Amrouche sur son activité à Tunisie française littéraire ${ }^{39}$. Tunisie française littéraire témoigne également d'une volonté de profiter des bouleversements du milieu littéraire français dus à la guerre pour tenter d'imposer Tunis comme un centre culturel de plein droit (tout comme s'y emploie sa consoeur Fontaine à Alger au même moment), à distance de la métropole et de cercles littéraires parisiens considérés comme compromis.

Lorsque Jean Amrouche, lassé du travail harassant (et effectué bénévolement) que lui demande Tunisie française littéraire depuis le départ de Guibert, jette l'éponge fin juin 1942, la page littéraire s'interrompt et laisse la place à La Tunisie française économique. La Tunisie française cessera de paraître en novembre 1942 (conséquence du débarquement allié en Afrique du Nord et de l'occupation allemande de la zone Sud). Elle ne reprendra pas pour autant en tant que telle en

33 P. Ricoeur, Sur la traduction, Les Belles Lettres, coll. «Traductologiques », Paris 2018, p. 35.

34 Ibidem.

35 Ibidem, p. 36.

36 A. Camus, «Lettre de France pour préparer le fruit », 25.01.1941.

37 M. Sauvage, « Sous les oliviers de l'Ermitage avec le Maréchal », 1.02.1941. Pour ne pas parler de P. Seghers qui évoque dans Tunisie française littéraire « l'homme de chez nous qui a sauvé le pays, nous l'aimons sans délire hurlant, mais avec application, avec une sombre et sévère ferveur » (idem, " L'époque “Contrast” », 28.12.1941).

${ }^{38}$ Cf. M. Corriou, op. cit., p. 516.

39 Cf. ibidem, p. 513. 
1944 : on y trouvera alors seulement, et de façon très irrégulière, quelques entrefilets littéraires intitulés « Défense de l’Esprit » (sur Johann Wolfgang von Goethe, Victor Hugo, la revue L'Arche, Vercors, l'art en général). En 1947, La Tunisie française sera remplacée par un nouveau titre, Tunisie-France. L'aventure littéraire de ce journal, la soif de poésie et l'effervescence intellectuelle dont il rendit compte dans ses pages durant les années de guerre, étaient, dans la France de la Libération confrontée bientôt à d'autres défis (notamment ceux de la décolonisation), désormais bel et bien révolues.

\author{
ANNEXE \\ PRINCIPAUX ARTICLES PARUS \\ DANS TUNISIE FRANÇAISE LITTÉRAIRE
}

\title{
1940 (à partir du 16.11)
}

16.11 «L'honneur d'écrire » (A. Guibert) ; « Procès des intellectuels » (J. Amrouche) ; « La France en marche. L'heure des poètes » (M. Sauvage)

23.11 « Patrice de la Tour du Pin et la Tunisie » (non signé) ; « Mission du poète » (M. Sauvage) ; «Cahiers du Sud» (A. Guibert)

30.11 «Qu'attendez-vous d'un écrivain?» (M. Sauvage) ; «Amitié franco-portugaise » (A. Guibert); « Procès des intellectuels. Prestige de l'action» (J. Amrouche)

7.12 «Procès des intellectuels. Qui veut sauver sa vie la perdra » (J. Amrouche)

14.12 « Sainteté de la poésie » (J. Amrouche) ; « Défense du rêve » (M. Sauvage) ; « Poésie 40 » (non signé)

28.12 « Tunis et la décentralisation littéraire. Regard sur le passé » (A. Guibert)

1941

4.01 « Tunis Capitale» (A. Guibert)

11.01 « Hommage à Bergson » (A. Patri)

18.01 « James Joyce» (A. Guibert)

25.01 «Lettre de France pour préparer le fruit» (A. Camus) ; «Fonction de la critique » (J. Amrouche)

1.02 « Dignité de la critique » (J. Amrouche) ; «L'honneur du poète » (F. Bonjean) ; « Sous les oliviers de l'Ermitage avec le Maréchal » (M. Sauvage)

8.02 « Indignités de la critique » (J. Amrouche) ; «L'honneur du poète» (F. Bonjean)

15.02 «Et la poésie aux poètes » (M. Sauvage)

1.03 « Psychologie du critique » (J. Amrouche); « Fontaine» (non signé)

8.03 « Liberté du poète » (A. Guibert) ; « France de chaque jour» (A. Borne)

15.03 «Lusitanie » (A. Guibert) ; « Ce qu'on ne peut pas dire» (P. Seghers) ; « Pour un nouvel ordre classique » (J. Amrouche)

22.03 «L'École de Paris devant la Révolution Nationale » (M. Sauvage)

29.03 « Une belle utopie — La poésie pour tous » (A. Guibert) 
5.04 «La poésie sera libre ou ne sera pas » (A. Guibert) ; « Pour une poésie africaine » (J. Amrouche)

19.04 « De la trahison et de la fidélité des clercs. Erasme et T. More» (M.P. Fouchet) ; «Pour une poésie africaine. Un drame spirituel : le bilinguisme» (J. Amrouche)

26.04 « Marxisme et freudisme » (G. Thibon) ; «Poésie et mystique » (G. Errache) ; « Pour une poésie africaine. L'Afrique aussi est une personne » (J. Amrouche)

3.05 « Poésie d'abord » (H. Bosco) ; "Pour une poésie africaine. Il viendra un jeune poète » (J. Amrouche)

16.05 « Présence d'un grand poète : P. Emmanuel » (J. Amrouche); « Poésie d'abord » (H. Bosco); «Peintres et sculpteurs en $1941 »$ (G. Brunon-Guardia)

17.05 « Confidences d'une race. L'Atlas sonore de la France» (M. Sauvage)

24.05 « Comme un feu d'étoupes » (A. Camus) ; « La libération spirituelle» (M. Sauvage) ; « Sur le mot mystique » (P. Emmanuel) ; « Malte » (A. Guibert)

7.06 « Islam et poésie. Le dhikre et la tourterelle » (F. Bonjean) ; « Malte» (A. Guibert)

14.06 « Rilke nous parle » (A. Guibert) ; «Claudel livré aux foules » (J. Amrouche)

28.06 « Les idées de la vieille France sur la colonisation » (M. Schveitzer, vice-recteur de l'Académie d'Alger) ; « Poèmes de Pierre Emmanuel » ; « Le premier livre d'un grand poète. Tombeau d'Orphée » (A. Guibert)

\section{Le journal s'interrompt les 11.07 et 16.08 .1941}

19.07 «L'unité spirituelle de l'Empire » (J. Amrouche) ; «Un témoin de notre temps » (A. Guibert) — sur Brasillach

2.08 « Poésie encore » (A. Guibert) ; « À propos du folklore berbère. L'invitation à la joie » (J. Amrouche)

9.09 « Péguy sans fard» (A. Guibert)

13.09 « La France retrouvée » (A. Guibert); « Barrès et notre adolescence » (J. Amrouche)

20.09 « Plume et charrue ou l'abus du roman paysan » (F. de Miomandre) ; « Regain de poésie » (A. Guibert)

27.09 « André Gaillard et la flamme des Cahiers du Sud» (A. Guibert) ; « Poésie et chanson populaire : trois chants de danse du pays Kabyle» (J. Amrouche)

4.10 « La poésie arabe citadine en Algérie » (M. Lacheraf) ; «Renaissance du génie d'Oc » (non signé)

11.10 «Un appel pour la gloire de Milosz » (A. Guibert) ; «Pyrénées, cahiers des lettres et des arts » (non signé) ; « Cuisine française » (G. Stein)

18.10 « Une nouvelle traduction de F. Garcia Lorca » (J. Amrouche)

25.10 « Arguments du poète » (J. de Boschère)

8.11 « Hommage à G. Apollinaire » (J. Amrouche) ; « Reconnaissance à L.P. Fargue » (F. de Miomandre); " Quatre Vents » (non signé)

15.11 «Rimbaud fils du soleil » (J. Amrouche)

22.11 « Raisons d'aimer la France » (A. Guibert) ; « Un grand poète de la terre créole : Daniel Thaly » (E. Flavia Leopold) ; « La Camargue et sa poésie. Le pays, le poète : Joseph d'Arbaud» (H. Bosco) (suite dans les $n^{\circ}$ du 29.11 et du 6.12); « La vie intellectuelle et artistique à Tunis » (J. Amrouche)

29.11 «Au balcon de l'Occident. Lisbonne retrouvée» (A. Guibert)

6.12 « Haute et basse littérature » (F. de Miomandre) ; « Mozart et les poètes » (H. Parrot)

20.12 « Beauté, mon beau souci» (A. Guibert)

27.12 « Le Maréchal Pétain. Paroles aux Français » (F. Bonjean) 
3.01 « Le Maréchal Pétain. Paroles aux Français » (F. Bonjean) (suite) ; « Chant pastoral berbère entendu dans le grand Atlas » (H. Bosco)

10.01 « Défense de la littérature française contemporaine » (L. Gillet de l'Académie française)

17.01 « Montherlant le cruel» (A. Guibert)

24.01 « P.J. Jouve, poète de l'angoisse humaine » (J. Amrouche)

31.01 « Souvenirs sur Félix Fénéon » (F. de Miomandre)

7.02 «Au secours de l'Occident. Message actuel de l'Inde. Cahiers du Sud, nº spécial» (J. Amrouche); «Prière sur le seuil » (poème de P. Emmanuel)

14.02 «Air de partout. Poésie sous quelques cieux» (I. de Franceschi)

21.02 « Des nouvelles de P. de la Tour du Pin » (poème) ; «Actualité des Troubadours. Pyrénées $\mathrm{n}^{\circ} 2 »$ (J. Amrouche)

28.02 « La sagesse de Goethe » (non signé)

7.03 « Le grand Majoral du Félibrige de la courtoisie française » (J. Amrouche) ; « In memoriam S. Zweig » (I. de Franceschi)

21.03 « Saint Stéphane Mallarmé » (J. Amrouche)

28.03 « Notre Bergson » (E. Flavia Leopold) ; « Notes pour servir à l'armée nouvelle. Grandeur du chant» (J. Roy)

4.04 «Esprit et vermine » (G. Germain)

25.04 « Gare de triage » (I. de Franceschi)

2.05 «La poésie de Jules Roy » (J. Amrouche) ; « Procès du surréalisme » (H. Monnier)

9.05 « Expulsons les monstres » (P. Seghers); «La poésie comme exercice spirituel » (non signé)

16.05 « J.J. Rousseau et le problème de l'homme» (E. Flavia Leopold) ; «André Gide et les jeunes poètes » (non signé)

23.05 « Lanza del Vasto » (J. Amrouche) ; « À propos de Péguy » (Agathocle)

30.05 « Islam et poésie. Conférence de F. Bonjean» (J. Amrouche)

6.06 « James Joyce, miroir d'une époque » (A. Guibert); « Monseigneur Bollon, prince de l'Église et homme de cœur» (J. Roy)

13.06 « Vocation de la Méditerranée » (G. Audisio); « Notes sur la création et le poète » (F. Bonjean)

20.06 « L'expression poétique et le pouvoir des mots » (E. Flavia Leopold) ; « Avec L. del Vasto, contemporain de Dante » (Perruchot)

27.06 «P. Emmanuel, témoin de notre temps » (J. Amrouche) ; « Image et beauté poétique » (E. Flavia Leopold)

\title{
NORTH AFRICA, A NEW FRENCH LITERARY CENTRE BETWEEN 1940 AND 1944? THE EXAMPLE OF TUNISIE FRANÇAISE LITTÉRAIRE IN THE MIRROR OF TRANSLATION
}

\begin{abstract}
The German Occupation of France (starting in summer 1940) brought about a brutal reclassification of literary values and a redefinition of "center" and "periphery" in the French Republic of Letters. The outcome of this phenomenon is particularly interesting in North Africa between 1940-1944. Indeed, the periodical Fontaine (edited in Algiers by Max Pol Fouchet), as well as Tunisie française littéraire (edited in Tunis under the aegis of Armand Guibert and Jean Amrouche), express a strong desire to take over a Parisian "center" discredited by the Occupation and the Collaboration, and create new "literary capitals" on the fringes of the metropolis. This paper focuses on Tunisie française littéraire (a very influential publication in North Africa during the war, to which
\end{abstract}


A. Camus and G. Stein contributed), analyses the role of cultural mediation played by literary journals geographically "peripheral" and their members in an attempt to redefine the contours of the "center" (Paris) and the "periphery" (the French colonial Empire) — an initiative where translations, particularly of indigenous authors, proved to be an important issue.

Key words: translation, poetry, France, German Occupation, periodicals, North Africa. 